\title{
Flavour Non-Universal UMSSM with Minimal Number of Exotics
}

\author{
Yaşar Hiçyılmaz, ${ }^{1,2}$ Stefano Moretti, ${ }^{2}$ and Levent Solmaz ${ }^{1}$ \\ ${ }^{1}$ Department of Physics, Balikesir University, TR10145, Balkesir, Turkey \\ ${ }^{2}$ School of Physics \& Astronomy, University of Southampton, Highfield, Southampton SO17 1BJ, UK
}

\begin{abstract}
We report the phenomenological implications of several family non-universal $U(1)^{\prime}$ sub-models in the $\mathrm{U}(1)^{\prime}$-extended Minimal Supersysmmetric Standard Model (UMSSM) possesing an extra down quark type exotic field. In doing this, we have started by enforcing anomaly cancellation criteria to generate a number of solutions in which the extra $\mathrm{U}(1)^{\prime}$ charges of the particles are treated as free parameters. We have then imposed existing bounds coming from colliders and astrophysical observations on the assumed submodels and observed that current limits dictate certain charge orientations.
\end{abstract}

Keywords: supersymmetry, flavour non-universality, exotics

DOI: 10.31526/ACP.BSM-2021.19

\section{INTRODUCTION}

Supersymmetry (SUSY) is one of the most studied NP theories at LHC, since it has remarkable advantages.

- The Hierarchy Problem.

- Natural light Higgs boson.

- Unification of the gauge couplings.

- WIMP candidate in order to solve the DM puzzle.

U(1) extended MSSMs (UMSSMs) have been broadly worked upon the literature, e.g. $E_{6}$ motivated, $B-L$ and Secluded UMSSMs. Such extensions can stem from Superstring and Grand Unified Theories (GUTs) [1, 2]. These models can dynamically generate the $\mu$ term at the EW scale by introducing new singlet Higgs field as $\mu=\frac{1}{2} \lambda\langle S\rangle[3,4,5]$. They also introduce a new massive neutral gauge boson, called $Z^{\prime}$ and $\mathrm{U}^{(1)^{\prime}}$ charges must satisfy the Anomaly Cancellation Conditions (ACCs) stemming from the additional $\mathrm{U}(1)^{\prime}$ group insertion.

ACC san be satisfied with two different ways.

- Adding exotics into the model.

- Using non-holomorphic terms without any exotics, called minimal UMSSM in which the $U(1)^{\prime}$ charges can be flavour nonuniversal.

A family non-universal $Z^{\prime}$ boson can explain the experimental results on $R_{K}$ and $R_{K^{*}}$ that deviate from SM [6]. One can find numerous well-motivated $\mathrm{U}(1)^{\prime}$ sub-models which give new and different signals for the detection of the extra $Z^{\prime}$.

In this report, we follow a hybrid approach, in which we will study a supersymmetric $\mathrm{U}(1)^{\prime}$ model with an additional exotic quark superfield, $\widehat{D_{x}}$ with non-universal $\mathrm{U}(1)^{\prime}$ charges in the lepton sector. We aim to find the possible charge configurations which satisfy not only the ACCs but also experimental low energy constraints. We also probe the evidence of the new $Z^{\prime}$ state precisely from its signals involving the other new features of the model, i.e., the coloured exotic states and the anomalous leptonic couplings.

\section{FAMILY NON-UNIVERSAL UMSSM}

The superpotential in the model allows Yukawa couplings for the quarks and third family leptons as well as couplings for the exotic fields given by

$$
\widehat{W}=h_{u} \widehat{Q} \cdot \widehat{H}_{u} \widehat{U}+h_{d} \widehat{Q} \cdot \widehat{H}_{d} \widehat{D}+h_{\tau} \widehat{L}_{3} \cdot \widehat{H}_{d} \widehat{E}_{3}+\lambda \widehat{S} \widehat{H}_{u} \cdot \widehat{H}_{d}+h_{v} \widehat{L} \cdot \widehat{H}_{u} \widehat{N}+\kappa \widehat{S} \widehat{D}_{x} \widehat{D}_{x},
$$

where $\hat{Q}$ and $\hat{L}_{3}$ denote the left-handed chiral superfields for the quarks and third family leptons while $\hat{U}, \hat{D}, \hat{E}_{3}$ and $\hat{N}$ stand for the right-handed chiral superfields of $u$-type quarks, $d$-type quarks, $\tau$-type leptons and neutrinos, respectively. Here, $H_{u}$ and $H_{d}$ are the MSSM Higgs doublets and $h_{u, d, \tau}$ are the Yukawa couplings to the matter fields. 
Our exotic particles have same charges as the down quarks. They are colour triplet and vector-like with respect to the MSSM, but chiral under the $U(1)^{\prime}$ symmetry. They only interact with $S$ and cannot mix with $S M$ fermions and to be expected having masses larger than $1 \mathrm{TeV}$. The mass of the exotic fermion can be written as follows:

$$
m_{D_{x}}=\frac{1}{\sqrt{2}} v_{S} \kappa
$$

Additionally, the mass squared matrix of the supersymmetric partners of the exotics can be written as follows:

$$
m_{\tilde{D}_{x}}^{2}=\left(\begin{array}{cc}
m_{\tilde{D}_{x}^{L}}^{2} & \frac{1}{2}\left(\sqrt{2} v_{S} T_{\mathcal{K}}-v_{d} v_{u} \lambda \kappa\right) \\
\frac{1}{2}\left(\sqrt{2} v_{S} T_{\kappa}-v_{d} v_{u} \lambda \kappa\right) & m_{\tilde{D}_{x}^{R}}^{2}
\end{array}\right),
$$

\section{RESULTS}

Fig. 1 shows the comparison of the experimental limits on the $Z^{\prime}$ boson mass and its cross section as obtained from direct searches in the processes $p p \rightarrow l l$ at $\mathcal{L}=137 \mathrm{fb}^{-1}$ [7] with full acceptance and $p p \rightarrow$ jets with experimental acceptance $A=0.5$ at $\mathcal{L}=137$ $\mathrm{fb}^{-1}$ [8] as well as $\mathcal{L}=27 \mathrm{fb}^{-1}$ and $36 \mathrm{fb}^{-1}$ [9]. We find that the $Z^{\prime}$ boson mass cannot be smaller than $4.5 \mathrm{TeV}$ in the light of the ATLAS di-lepton results [7] and the ATLAS results on the $Z^{\prime} \rightarrow$ jets channel does not put any further limit on the $Z^{\prime}$ mass, as in the right panel we have additionally applied the $Z^{\prime}$ mass constraint from the left panel.
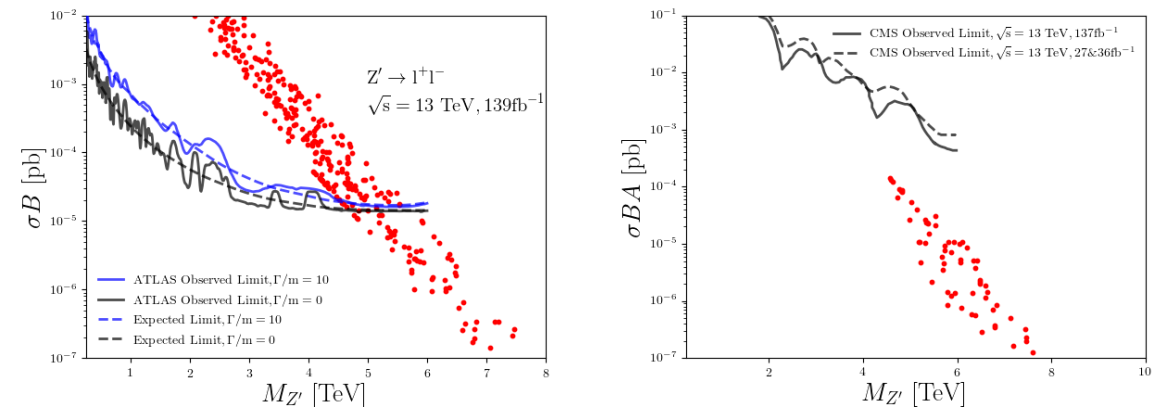

FIGURE 1: The $Z^{\prime}$ boson mass limits on $\sigma\left(p p \rightarrow Z^{\prime} \rightarrow l l\right) v s M_{Z^{\prime}}$ (left panel) and $\sigma\left(p p \rightarrow Z^{\prime} \rightarrow\right.$ jets) vs $M_{Z^{\prime}}$ (right panel), where $l$ describes electron and muon while jets refer to contributions from both the $S M$ and exotic quarks. All points plotted here satisfy all experimental constraints given in the previous section. In the right panel, we have additionally applied the $Z^{\prime}$ mass constraint from the left panel.

Fig. 2 depicts the $\mathrm{U}(1)^{\prime}$ charge sets satisfying various theoretical and experimental bounds. The gray points correspond to configurations compliant with ACCs, REWSB and a neutralino as the Lightest Supersymmetric Particle (LSP). The green points form a subset of the gray ones as they also satisfy all the experimental constraints, i.e., the exotic mass limits, the constraints from the rare $B$-decays and the bound on the relic abundance of the neutralino LSP. Red points instead form a subset of the green ones as they are also compatible with the $Z^{\prime}$ boson mass bounds from direct searches shown in Fig. 1 . When ACCs and all constraints are applied, charges are restricted to certain regions, e.g., the $-0.5 \lesssim Q_{H_{u}}, Q_{H_{d}} \lesssim 0.5$ domain is favoured. Also, the lower limit on $\left|Q_{S}\right|$ should be $\sim 0.4$ and ACCs allow the $Q_{Q}$ charge to be in a narrow interval, as $-0.25 \lesssim Q_{Q} \lesssim 0.25$.

We show the particle mass spectrum in Fig 3. Stop and sbottom masses are heavy in general and one can extract a $\sim 3 \mathrm{TeV}$ lower mass limit. On the other hand, the mass of the exotic quarks can be as light as $1.1 \mathrm{TeV}$, as the exotic scalar masses should be larger than $2.5 \mathrm{TeV}$. The LSP neutralino is relatively heavy as $1 \mathrm{TeV} \lesssim m_{\tilde{\chi}_{1}^{0}} \lesssim 1.3 \mathrm{TeV}$. Moreover, due to relic density constraints, the lightest neutralino and lightest chargino are very degenerate in mass and the LSP neutralino probably are Higgsino-like. 

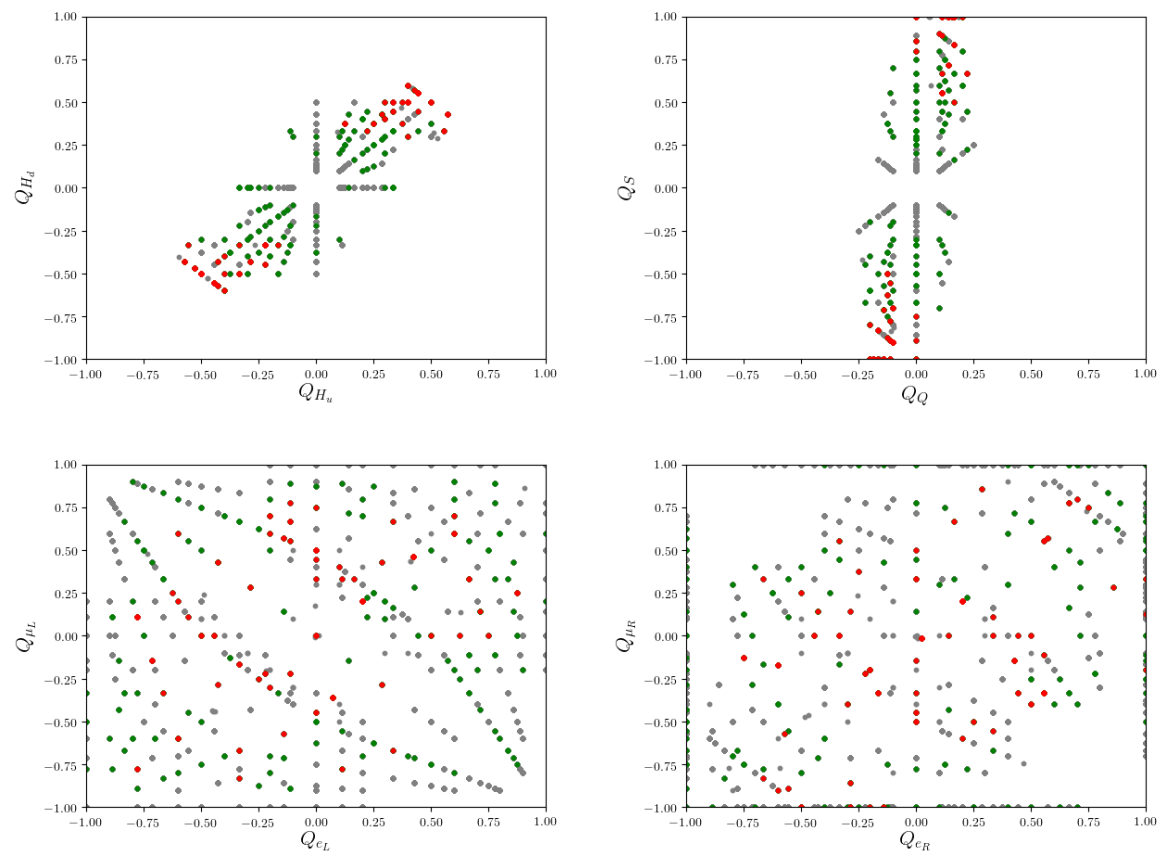

FIGURE 2: The distributions of the $U(1)^{\prime}$ charges allowed by various theoretical and experimental conditions over the following planes: $Q_{H_{u}}-Q_{H_{d^{\prime}}}$ $Q_{Q}-Q_{S}, Q_{e_{L}}-Q_{\mu_{L}}$ and $Q_{e_{R}}-Q_{\mu_{R}}$. All points are consistent with REWSB, ACCs and neutralino LSP. Green points are a subset of the gray ones as they also satisfy all experimental constraints. Red points are a subset of the green ones as they are also compatible with the $Z^{\prime}$ boson mass bounds in Fig. 1.
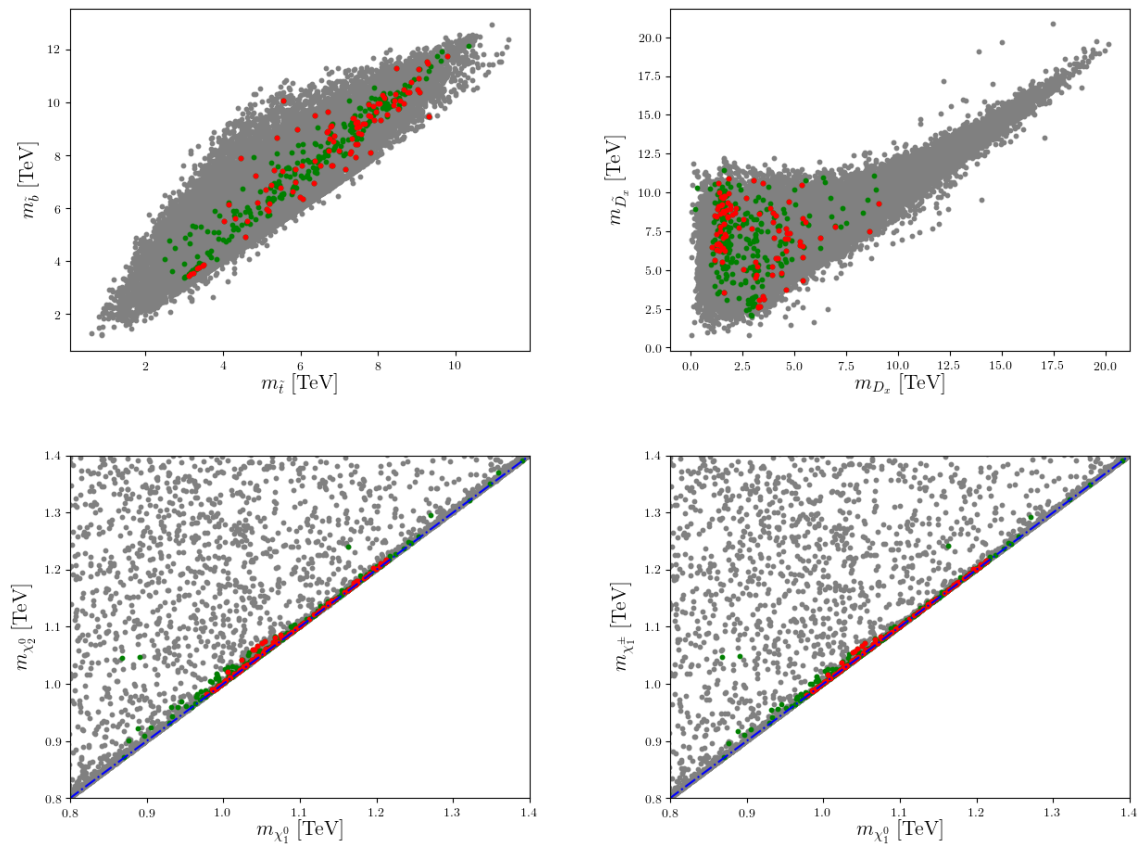

FIGURE 3: The mass spectrum of the lightest chargino, two lightest neutralinos, stops, sbottoms and exotic (both fermionic and scalar) states over the following planes: $m_{\tilde{t}}-m_{\tilde{b}}, m_{D_{x}}-m_{\tilde{D}_{x^{\prime}}}, m_{\tilde{\chi}_{1}^{0}}-m_{\tilde{\chi}_{2}^{0}}$ and $m_{\tilde{\chi}_{1}^{0}}-m_{\tilde{\chi}_{1}^{ \pm}}$. The colour convention is the same as in Fig. 2.

\section{CONCLUSIONS}

In this report, we have studied the implications of flavour non-universal $\mathrm{U}(1)^{\prime}$ charges with exotic coloured states, fermionic and scalar. We implementi both theoretical and experimental constraints such as ACCs, REWSB, yielding a neutralino as the LSP, relic density, $B$-physics and collider bounds. While the theoretical conditions imposed allows for a symmetric distribution of such charges, wherein $Q_{H_{u}} \sim Q_{H_{d}}$ is preferred and $Q_{S}$ cannot be very small (these are the doublet and singlet charges), the enforcement of the experimental ones renders the eventual solutions significantly different with a noticeable loss of symmetry. Nonetheless, the 
surviving charges produce UMSSM configurations inducing phenomenological implications that could be probed at high energy accelerators presently discussed as successors to the LHC.

\section{ACKNOWLEDGMENTS}

SM is supported in part through the NExT Institute and the STFC Consolidated Grant No. ST/L000296/1. The work of YH is supported by The Scientific and Technological Research Council of Turkey (TUBITAK) in the framework of 2219-International Postdoctoral Research Fellowship Program.

\section{References}

[1] M. Cvetic and P. Langacker, Mod. Phys. Lett. A 11 (1996) 1247-1262 [hep-ph/9602424].

[2] J. L. Hewett and T. G. Rizzo, Phys. Rept. 183 (1989) 193.

[3] D. Suematsu and Y. Yamagishi, Int. J. Mod. Phys. A 10 (1995) 4521-4536 [hep-ph/9411239].

[4] V. Jain and R. Shrock, [hep-ph/9507238.]

[5] Y. Nir, Phys. Lett. B 354 (1995) 107-110 [hep-ph/9504312].

[6] R. Aaij et al. [LHCb collaboration], Phys. Rev.Lett. 113 (2014) 151601 [arxiv:1406.6482].

[7] G. Aad et al. [ATLAS collaboration], Phys. Lett. B 796 (2019) 68-87 [arxiv : 1903 . 06248].

[8] A. M. Sirunyan et al. [CMS collaboration], JHEP 05 (2020) 033 [arxiv: 1911 . 03947].

[9] A. M. Sirunyan et al. [CMS collaboration], JHEP 08 (2018) 130, [arxiv: 1806. 00843]. 